Original paper

\title{
Comparison of knowledge and awareness between consultant physicians and resident doctors about non-alcoholic fatty liver disease
}

\author{
Anurag Shrikant Lavekar', Aditi Deshpande², Dhananjay Raje ${ }^{3}$ \\ 1JSS Hospital, Karnataka, India \\ ¿Lokmanya Tilak Municipal Medical College and General Hospital, Mumbai, India \\ ${ }^{3}$ MDS Bio-Analytics Private Ltd., Nagpur, India
}

\begin{abstract}
Aim of the study: Non-alcoholic fatty liver disease (NAFLD) is a multisystem disease and is commonly associated with diabetes mellitus, dyslipidaemia, hypertension and obesity. These illnesses are usually treated by physicians, and hence they need to stay updated on NAFLD. The aim of the study was to assess and compare the knowledge and awareness about NAFLD among consultant physicians and resident doctors.

Material and methods: A questionnaire concerning epidemiology, risk factors, complications, diagnostic methods, management options, progression and screening of NAFLD was given to the consultant physicians and resident doctors and their responses were sought. The comparison of responses was carried out between residents and consultants using Pearson's $\chi^{2}$ test.

Results: A total of 240 doctors participated in the study with 60 resident doctors and 180 consultant physicians. $45 \%$ of the total participants did not consider NAFLD as a major health hazard. Consultants had better knowledge than residents about the prevalence of NAFLD, and the risks due to various factors. Also they had better knowledge about non-invasive diagnostic modalities. Resident doctors advocated use of antioxidants more than consultants. There was no statistically significant difference of perception between residents and physicians about association of NAFLD with diabetes and obesity, diet advice, dietary modification and exercise, usage of medications, avoidance of hepatotoxic drugs and alcohol.

Conclusions: This study revealed that physicians participating in our survey appreciate the prevalence of NAFLD but are unaware of the seriousness and the optimal management. This has implications for targeting 'at-risk' populations and appropriate referral of patients to gastroenterology/hepatology clinics.
\end{abstract}

Key words: knowledge, non-alcoholic fatty liver disease, residents, consultants.

Address for correspondence:

Dr. Anurag Shrikant Lavekar, JSS Hospital, Karnataka, India, e-mail: anuraglavekar@gmail.com

\section{Introduction}

Non-alcoholic fatty liver disease (NAFLD) is a spectrum of diseases that ranges from simple steatosis to steatohepatitis (NASH) to fibrosis and cirrhosis. The risk of progression from steatosis to steatohepatitis is as high as $44-64 \%$ and risk of progression to advanced fibrosis is $24 \%[1,2]$. In a small proportion of cases, NAFLD can lead to serious complications including decompensated cirrhosis, liver failure and hepa- tocellular carcinoma [3]. NAFLD increases the risk of development of hepatocellular carcinoma by $9 \%$ every year [4].

Non-alcoholic fatty liver disease has a multidirectional relationship with the metabolic syndrome $[5,6]$. The standard definition put forth by the European Association for the Study of the Liver in the current practice guidelines is "NAFLD is characterised by excessive hepatic fat accumulation, associated with insulin resistance (IR), and defined by the presence of steatosis in 
$>5 \%$ of hepatocytes according to histological analysis or by the proton density fat fraction (PDFF, providing a rough estimation of the volume fraction of fatty material in the liver) $>5.6 \%$ assessed by proton magnetic resonance spectroscopy ( $\left.{ }^{1} \mathrm{H}-\mathrm{MRS}\right)$ or quantitative fat/ water selective magnetic resonance imaging (MRI)" [7]. Published literature demonstrates its association with increased risk of cardiovascular disease $[8,9]$ and cancer [10]. NAFLD also shows increased risk of micro- and macrovascular complications as in people with diabetes $[11,12]$. All these complications place these patients at two-fold risk of all-cause mortality [13]. Earlier, NAFLD was thought to be a disease of affluent nations, but recent studies indicate that it is equally prevalent in developing countries such as India [14].

With the alarming increase in the prevalence of non-communicable diseases such as diabetes mellitus, hypertension, obesity, dyslipidaemia, etc., the prevalence of NAFLD is also reaching epidemic levels [15]. It has become the most common cause of liver diseases worldwide [16]. The precise prevalence of NAFLD is difficult to assess as it varies geographically and differs in different ethnicities. It is estimated to be $32 \%$ in the Middle East and $27 \%$ in Asia. In India, the prevalence of NAFLD is reported to vary from rural to urban populations owing to the economic divide [16]. In the urban Indian population NAFLD is reported to have a prevalence between 16 and 32\%, whereas in rural India it is reported to be $9 \%[14,17,18]$. Contrary to this, another study reports NAFLD prevalence of $28.1 \%$ in rural India [19].

Despite the serious long-term effects associated with NAFLD, the awareness about NAFLD in the general population is low $[20,21]$. NAFLD is commonly associated with diabetes mellitus, dyslipidaemia, hypertension and obesity. These defining illnesses are usually treated by physicians. Given the geographic and racial differences, complex pathogenesis, subtle initial presentation, involvement of multiple organ systems, complications, intricacies in evolving diagnostic methods and cost of management, it is important for referring physicians to be well aware of this initially benign appearing and rapidly growing disease in its early phase. Hence, we decided to assess the knowledge and awareness about NAFLD among physicians.

\section{Material and methods}

A comprehensive questionnaire detailing factors associated with NAFLD was given to the resident doctors (post-graduates students in internal medicine) and consultant physicians (practising doctors holding an $\mathrm{MD} / \mathrm{DNB}$ in internal medicine) in Bangalore city.
A similar questionnaire has been used previously for assessing knowledge about NAFLD in doctors in Sri Lanka [22]. The first part of the questionnaire comprised demographic variables such as name, age, gender, resident doctor or consultant physician. The second part consisted of questions assessing knowledge of risk factors, complications, methods of diagnosis, management options, progression and screening of NAFLD. The study was approved by the Institutional Ethical Committee.

\section{Statistical methods}

The responses of participants to different questions related to awareness, treatment and management were obtained and summarized in terms of frequency and percentage. The participants were categorized into two groups, namely, resident doctors and consultant physicians. All the responses were compared between these two groups based on frequencies using Pearson's $\chi^{2}$ test. The consultant physicians were further subdivided into junior consultant physicians ( $\leq 40$ years) and senior consultant physicians ( $>40$ years) for comparisons based on experience. All the computations were carried out using SPSS ver. 20.0 (IBM Corp. ARMONK USA) software and the statistical significance was evaluated at the $5 \%$ level.

\section{Results}

Table 1 provides descriptive statistics for participant characteristics. There were 180 (75\%) consultants and 60 (25\%) resident doctors who participated in the study. Amongst these, 187 (77.9\%) were male and $53(22.1 \%)$ were female. As regards age distribution, $62(25.8 \%)$ were less than 30 years of age, while $59(24.6 \%)$ were above 45 years and $96(40.1 \%)$ were in the range of $36-45$ years. The mean of participants was $42.05 \pm 10.12$ years. Table 2 shows the awareness in participants about different aspects of NAFLD.

As regards epidemiology, 132 (55\%) opined that NAFLD is a major health problem. The majority, i.e. 110 (45.8\%), believed that the prevalence of NAFLD ranges between 31 and 40\%, while 60 (25\%) believed it to be in the range of $21-30 \%$. The difference in perception among resident doctors and consultants about the prevalence was significantly different $(p<0.0001)$. The consultants considered the prevalence to be greater than that stated by residents. Both groups equally advocated use of low lipid content diet (45.4\%) and hypocaloric diet (43.3\%). 
Table 1. Descriptive statistics for participants

\begin{tabular}{lcc}
\hline Parameters & Level & Statistic \\
\hline Designation of participants, $n(\%)$ & Resident doctors & $60(25)$ \\
\cline { 2 - 3 } & Consultants & $180(75)$ \\
\hline Sex, $n(\%)$ & Female & $53(22.1)$ \\
\cline { 2 - 3 } & Male & $187(77.9)$ \\
\hline Age in years, $n(\%)$ & $<30$ & $62(25.8)$ \\
\cline { 2 - 3 } & $31-35$ & $23(9.6)$ \\
\cline { 2 - 3 } & $36-40$ & $51(21.3)$ \\
\cline { 2 - 3 } & $41-45$ & $45(18.8)$ \\
\hline Participant's age in years, mean $\pm S D$ & $>45$ & $59(24.6)$ \\
\hline
\end{tabular}

In the causation, $155(64.6 \%)$ participants opined that the "disease is inherited"; there was no statistically significant difference between the two groups.

Regarding associated disorders, all the participants $(100 \%)$ stated that diabetes and obesity are risk factors and therefore associated with NAFLD. The occurrence of hypertension along with NAFLD was experienced by $229(95.4 \%)$ respondents; however, the perception significantly differed between resident doctors $(83.3 \%)$ and consultants $99.4 \%(p<0.0001)$. The prevalence of dyslipidaemia with NAFLD was suggested by $98.9 \%$ of consultants, compared to $91.7 \%$ of residents (91.7\%) $(p=0.004)$. Further, $60.6 \%$ of the consultants opined that sleep apnoea accompanies NAFLD, and this pro-

Table 2. Awareness about different aspects of non-alcoholic fatty liver disease among resident doctors and consultants

\begin{tabular}{|c|c|c|c|c|c|}
\hline Parameters & Response & Resident doctors ( $n=60$ ) & Consultants $(n=180)$ & Total $(N=240)$ & $P$-value \\
\hline \multicolumn{6}{|c|}{ Awareness about epidemiology of NAFLD, $n(\%)$} \\
\hline NAFLD is a major problem & Yes & $28(46.7)$ & $104(57.8)$ & $132(55)$ & 0.134 \\
\hline \multirow[t]{5}{*}{ Prevalence of NAFLD (\%) } & $\leqslant 10$ & $7(11.7)$ & $3(1.7)$ & $10(4.2)$ & \multirow[t]{5}{*}{$<0.0001$} \\
\hline & $11-20$ & $10(16.7)$ & $9(5.0)$ & $19(7.9)$ & \\
\hline & $21-30$ & $16(26.7)$ & $44(24.4)$ & $60(25)$ & \\
\hline & $31-40$ & $23(38.3)$ & $87(48.3)$ & $110(45.8)$ & \\
\hline & $41+$ & $4(6.7)$ & $37(20.6)$ & $41(17.1)$ & \\
\hline Inherited & TRUE & $43(71.7)$ & $112(62.2)$ & $155(64.6)$ & 0.185 \\
\hline \multirow[t]{3}{*}{ Dietary management } & Low lipid content diet (Yes) & $26(43.3)$ & $83(46.1)$ & $109(45.4)$ & \multirow[t]{3}{*}{0.932} \\
\hline & Hypocaloric diet (Yes) & $27(45.0)$ & $77(42.8)$ & $104(43.3)$ & \\
\hline & $\begin{array}{l}\text { Low carbohydrate content } \\
\text { diet (Yes) }\end{array}$ & $7(11.7)$ & $20(11.1)$ & $27(11.3)$ & \\
\hline Is family screening recommended? & Yes & $4(6.8)$ & $30(16.8)$ & $34(14.3)$ & \\
\hline \multicolumn{6}{|c|}{ Awareness about associated disorders, $n(\%)$} \\
\hline DM & Yes & $60(100)$ & $180(100)$ & $240(100)$ & - \\
\hline HPT & Yes & $50(83.3)$ & $179(99.4)$ & $229(95.4)$ & $<0.0001$ \\
\hline Obesity & Yes & $60(100)$ & $180(100)$ & $240(100)$ & - \\
\hline Dyslipidaemia & Yes & $55(91.7)$ & $178(98.9)$ & $233(97.1)$ & 0.004 \\
\hline Apnoea & Yes & $14(23.3)$ & $109(60.6)$ & $123(51.3)$ & $<0.0001$ \\
\hline Hypothyroidism & Yes & $8(13.3)$ & $88(48.9)$ & $96(40)$ & $<0.0001$ \\
\hline PCOS & Yes & $8(13.3)$ & $65(36.1)$ & $73(30.4)$ & 0.001 \\
\hline IHD & Yes & $23(38.3)$ & $96(53.3)$ & $119(49.6)$ & 0.044 \\
\hline \multicolumn{6}{|c|}{ Awareness about diagnostic methods, $n(\%)$} \\
\hline USG & Yes & $47(78.3)$ & $175(97.2)$ & $222(92.5)$ & $<0.0001$ \\
\hline Thromboelastometry & Yes & $12(20.0)$ & $38(21.1)$ & $50(20.8)$ & 0.854 \\
\hline MRI & Yes & $5(8.3)$ & $95(52.8)$ & $100(41.7)$ & $<0.0001$ \\
\hline Liver biopsy & Yes & $50(83.3)$ & $162(90.0)$ & $212(88.3)$ & 0.164 \\
\hline Liver enzymes & Yes & 49 (81.7) & 170 (94.4) & $219(91.3)$ & $<0.0001$ \\
\hline
\end{tabular}

$P$-values in bold indicate statistical significance.

NAFLD - non-alcoholic fatty liver disease, DM - diabetes mellitus, HPT - hypertension, PCOS - polycystic ovary syndrome, IHD - ischemic heart disease, USG - ultrasonography, MRI - magnetic resonance 
portion was significantly higher than that of residents $(23.3 \%)(p<0.0001)$. Hypothyroidism, polycystic ovary syndrome (PCOS) and ischemic heart disease (IHD) were also believed by the consultants to accompany NAFLD, and the proportion of belief was significantly higher than that of the residents, with $p$-values $<0.0001,0.001$ and 0.044 respectively. Table 2 also shows the awareness about the diagnostic modalities. The perception about using ultrasonography (USG) of the abdomen and MRI differed significantly between the residents and the consultants. About 97.2\% of consultants recommended USG for diagnosis, and this proportion was significantly higher than that of the residents $(78.3 \%)(p<0.0001)$. Also, nearly $52.8 \%$ of consultants recommended MRI for diagnosis, and this proportion was significantly higher than that of the residents $(8.3 \%)(p<0.0001)$. The diagnosis based on liver enzymes was recommended strongly by the consultants as compared to the residents $(p<0.0001)$. Thromboelastometry (20.8\%) and liver biopsy $(88.3 \%)$ were advocated by both groups without a statistically significant difference. Table 3 shows the perception of participants about management, pharmacological treatment and barriers in the management. There was almost an absolute consensus (99.6\%) on dietary modification and exercise by all the respondents. Usage of vitamin $\mathrm{E}$ for nondiabetic patients (34.2\%), pioglitazones $(25 \%)$, statins $(42.9 \%)$ and avoidance of more than 7 units of alcohol (91.3\%) and hepatotoxic drugs (60.8\%) were advocated by both the groups without any statistically significant difference. The residents primarily preferred the use of antioxidants (68.9\%) as against consultants (51.7\%) and the difference in the proportions was statistically significant $(p=0.016)$. As regards the use of obeticholic acid, $17.2 \%$ of consultants were aware of this option compared to only $1.7 \%$

Table 3. Perception of resident doctors and consultants about management option, pharmacological treatment and barriers for management

\begin{tabular}{|c|c|c|c|c|c|}
\hline Parameters & Response & $\begin{array}{l}\text { Resident doctors } \\
\quad(n=60)\end{array}$ & $\begin{array}{l}\text { Consultants } \\
(n=180)\end{array}$ & $\begin{array}{c}\text { Total } \\
(N=240)\end{array}$ & $P$-value \\
\hline \multicolumn{6}{|l|}{ Management options, $n(\%)$} \\
\hline Dietary modifications & Yes & $59(98.3)$ & $180(100)$ & $239(99.6)$ & 0.083 \\
\hline Exercise & Yes & $59(98.3)$ & $180(100)$ & $239(99.6)$ & 0.083 \\
\hline Vitamin E for non-diabetic patients & Yes & $21(35.0)$ & $61(33.9)$ & $82(34.2)$ & 0.875 \\
\hline Pioglitazones & Yes & $15(25.0)$ & $45(25.0)$ & $60(25)$ & 0.999 \\
\hline Avoiding hepatotoxic drugs & Yes & $35(58.3)$ & $111(61.7)$ & $146(60.8)$ & 0.647 \\
\hline UDCA & Yes & 0 & 0 & 0 & - \\
\hline Avoiding > $7 \mathrm{U}$ of alcohol & Yes & $52(86.7)$ & $167(92.8)$ & $219(91.3)$ & 0.147 \\
\hline Statins & Yes & $23(38.3)$ & $80(44.4)$ & $103(42.9)$ & 0.408 \\
\hline Antioxidants & Yes & $31(51.7)$ & $124(68.9)$ & $155(64.6)$ & 0.016 \\
\hline Obeticholic acid & Yes & $1(1.7)$ & $31(17.2)$ & $32(13.3)$ & 0.002 \\
\hline Weight loss of 3-5\% & Yes & $16(26.7)$ & $103(57.2)$ & $119(49.6)$ & $<0.0001$ \\
\hline Weight loss of $10 \%$ & Yes & $10(16.7)$ & $91(50.6)$ & $101(42.1)$ & $<0.0001$ \\
\hline Referral to gastroenterologist & Yes & $5(8.3)$ & $36(20.0)$ & $41(17.1)$ & 0.037 \\
\hline \multicolumn{6}{|l|}{ Pharmacological treatment, $n(\%)$} \\
\hline Recommend statins for high AST and ALT & Yes & $0(0.0$ & $11(6.1)$ & $11(4.6)$ & 0.051 \\
\hline Recommend vitamin E for patient of NAFLD & Yes & $2(3.3)$ & $22(12.2)$ & $24(10.0)$ & 0.047 \\
\hline Aware of side effects of vitamin $E$ & Yes & $1(1.7)$ & $17(9.4)$ & $18(7.5)$ & 0.048 \\
\hline \multicolumn{6}{|l|}{ Barriers for management, $n(\%)$} \\
\hline Time constrain & Yes & $30(50.0)$ & $51(28.3)$ & $81(33.8)$ & 0.002 \\
\hline Lack of confidence in management & Yes & $44(73.3)$ & $159(88.3)$ & $203(84.6)$ & 0.005 \\
\hline Cost of evaluation and treatment & Yes & $38(63.3)$ & $121(67.2)$ & 159(66.3) & 0.581 \\
\hline Lack of compliance by the patient & Yes & $3(5.0)$ & $18(10.0)$ & $21(8.8)$ & 0.235 \\
\hline Uncomfortable to discuss obesity with patient & Yes & $0(0.0)$ & $1(0.6)$ & $1(0.4)$ & 0.563 \\
\hline
\end{tabular}

$P$-values in bold indicate statistical significance.

UDCA - ursodeoxycholic acid, AST - alanine aminotransferase, AST - aspartate aminotransferase, NAFLD - non-alcoholic fatty liver disease 
Table 4. Perception of consultants stratified by age about management option, pharmacological treatment and barriers for management

\begin{tabular}{|c|c|c|c|c|c|}
\hline Parameters & Response & $\begin{array}{c}\text { Age } \leqslant 40 \text { years } \\
(n=75)\end{array}$ & $\begin{array}{c}\text { Age }>40 \text { years } \\
(n=105)\end{array}$ & $\begin{array}{c}\text { Total } \\
(N=180)\end{array}$ & $P$-value \\
\hline \multicolumn{6}{|l|}{ Management options, $n(\%)$} \\
\hline Dietary modifications & Yes & $75(100)$ & $105(100)$ & $180(100)$ & - \\
\hline Exercise & Yes & $75(100)$ & $105(100)$ & $180(100)$ & - \\
\hline Vitamin E for diabetic patients & Yes & $19(25.3)$ & $42(40.0)$ & $61(33.9)$ & 0.041 \\
\hline Pioglitazones & Yes & $14(18.7)$ & $31(29.5)$ & $45(25.0)$ & 0.097 \\
\hline Avoiding hepatotoxic drugs & Yes & $39(52.0)$ & $72(68.6)$ & $111(61.7)$ & 0.024 \\
\hline UDCA & Yes & 0 & 0 & 0 & \\
\hline Avoiding $>7 \mathrm{U}$ of alcohol & Yes & $74(98.7)$ & $93(88.6)$ & $177(98.3)$ & 0.011 \\
\hline Statins & Yes & $34(45.3)$ & $46(43.8)$ & $80(44.4)$ & 0.839 \\
\hline Antioxidants & Yes & $48(64.0)$ & $76(72.4)$ & $124(68.9)$ & 0.231 \\
\hline Obeticholic acid & Yes & $13(17.3)$ & $18(17.1)$ & $31(17.2)$ & 0.973 \\
\hline Weight loss of 3-5\% & Yes & $40(53.3)$ & $63(60.0)$ & $103(57.2)$ & 0.373 \\
\hline Weight loss of $10 \%$ & Yes & $35(46.7)$ & $56(53.3)$ & $91(50.5)$ & 0.378 \\
\hline Referral to gastroenterologist & Yes & $18(13.3)$ & $23(21.9)$ & $41(22.8)$ & 0.081 \\
\hline \multicolumn{6}{|l|}{ Pharmacological treatment, $n(\%)$} \\
\hline Recommend statins for high AST and ALT & Yes & $6(8.0)$ & $5(4.8)$ & $11(6.1)$ & 0.371 \\
\hline Recommend vitamin E for patient of NAFLD & Yes & $6(8.0)$ & $16(15.2)$ & $22(12.2)$ & 0.144 \\
\hline Aware of side effects of vitamin $E$ & Yes & $3(4.0)$ & $14(13.3)$ & $17(9.4)$ & 0.035 \\
\hline \multicolumn{6}{|l|}{ Barriers for management, $n(\%)$} \\
\hline Time constraint & Yes & $19(25.3)$ & $32(30.5)$ & $51(28.3)$ & 0.451 \\
\hline Lack of confidence in management & Yes & $69(92.0)$ & $90(85.7)$ & $159(88.3)$ & 0.195 \\
\hline Cost of evaluation and treatment & Yes & $44(58.7)$ & $77(73.3)$ & $121(67.2)$ & 0.039 \\
\hline Lack of compliance by the patient & Yes & $4(5.3)$ & $14(13.3)$ & $18(10.0)$ & 0.078 \\
\hline Uncomfortable to discuss obesity with patient & Yes & $0(0.0)$ & $1(0.9)$ & $1(0.5)$ & 0.397 \\
\hline
\end{tabular}

P-values in bold indicate statistical significance.

UDCA - ursodeoxycholic acid, AST - alanine aminotransferase, AST - aspartate aminotransferase, NAFLD - non-alcoholic fatty liver disease

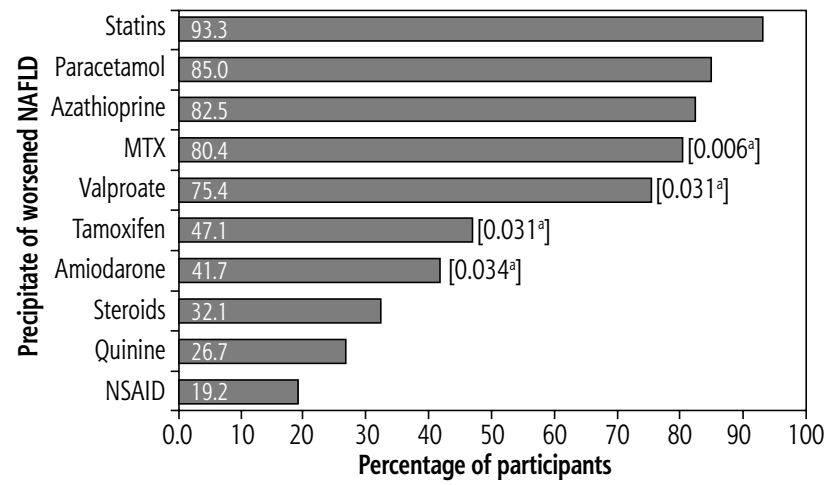

Fig. 1. Horizontal bar chart showing percentage of participants agreeing to the worsening effect of different agents/medications. A comparison between resident doctors and consultants

of the residents $(p=0.002)$. The weight loss of $3-5 \%$ was suggested by $57.2 \%$ of the consultants for NAFLD management, while only $26.7 \%$ of residents believed in the benefit of weight loss $(p<0.0001)$. In addition, the weight loss of $10 \%$ was suggested by $50.6 \%$ of the consultants for NAFLD management, while only $16.7 \%$ of residents believed in this weight loss $(p<0.0001)$. A significantly higher proportion of consultants $(20 \%)$ refer patients to gastroenterologists, as compared to residents $(8.3 \%)(p=0.037)$.

As regards treatment, the proportion of respondents recommending statins for high alanine aminotransferase (ALT) and aspartate aminotransferase (AST), as well as vitamin E for patients of NAFLD, was low, i.e. $4.6 \%$ and $10 \%$ respectively. Moreover, the proportion of consultants (12.2\%) recommending vitamin $\mathrm{E}$ was higher than residents (3.3\%), which was significant $(p=0.047)$. Consultants significantly had more knowledge about the side effects of vitamin $\mathrm{E}$ than residents $(9.4 \%$ vs. $1.7 \%)$ $(p=0.048)$. 


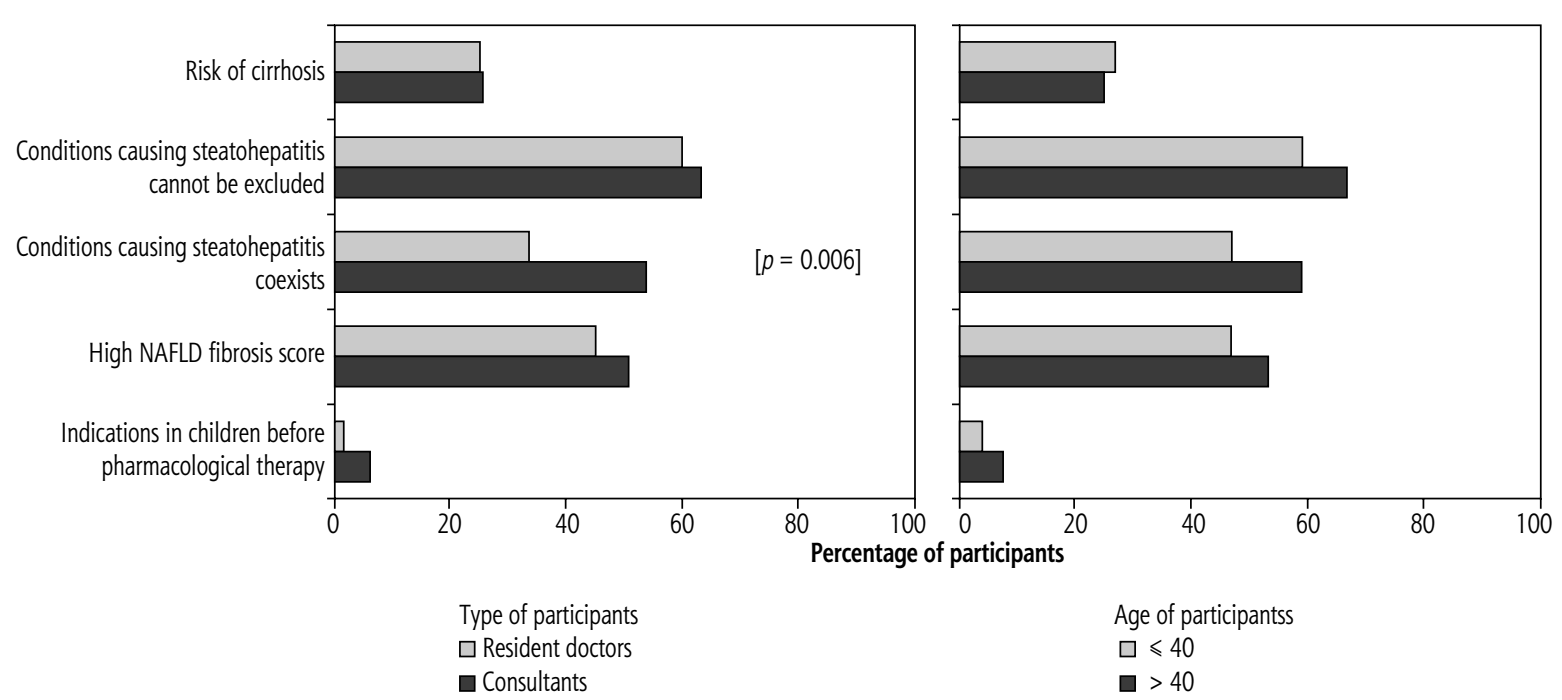

Fig. 2. Horizontal bar chart showing percentage of participants giving opinion on various indications of liver biopsy in study groups

Amongst the barriers in the management, about $33.8 \%$ of participants had concern about time management, residents having concern significantly more frequently (50\%) than consultants $(28.3 \%)(p=0.002)$. Another important barrier was the lack of confidence in management. Nearly $84.6 \%$ of participants had this barrier, which was expressed significantly more often by consultants $(88.3 \%)$ as compared to residents $(73.3 \%)(p=0.005)$. Cost of evaluation and treatment was another barrier experienced by the participants (66.3\%) without a significant difference between the two groups. Lack of compliance by the patient was not a major barrier as reported by both the groups (8.8\%).

The perception about management options, pharmacological treatment and barriers of management among junior and senior consultant physicians was analysed as shown in Table 4 . There was a statistically significant difference regarding the administration of vitamin $\mathrm{E}$ to nondiabetic patients among participants with age less than 40 years $(25.3 \%)$ and those above 40 years $(40 \%)(p=0.041)$. Further, there was a significant difference of opinion about avoiding hepatotoxic drugs between these two age categories $(p=0.024)$. The senior consultants avoid the use of such drugs $(68.6 \%)$ as against the junior consultants (52\%). Avoiding more than 7 units of alcohol per week was advised majorly by junior consultants (98.7\%) as against senior consultants $(88.6 \%)(p=0.011)$. There was concern about the cost of treatment among consultants. The rate of concern was significantly higher among senior consultants (73.3\%) than junior consultants (58.7\%) $(p=0.039)$.

Figure 1 gives the percentage of participants believing in the precipitating or worsening effect on NAFLD due to the usage of different drugs. The ma- jority (93.3\%) believe that statins have a worsening effect on NAFLD, followed by $85 \%$ who believe that paracetamol and $82.5 \%$ who believe that azathioprine have a worsening effect on NAFLD. Regarding usage of methotrexate (MTX), a significantly higher proportion of consultants (84.4\%) believed in its worsening effect, as against $68.3 \%$ of resident doctors $(p=0.006)$. Similarly, a high proportion of consultants believed that the use of valproate, tamoxifen, and amiodarone worsens NAFLD, as compared to resident doctors with proportions $78.9 \%$ vs. $65 \%, 51.1 \%$ vs. $35 \%$, and $45.6 \%$ vs. $30 \%$, respectively, all statistically significant.

Figure 2 shows the percentage of participants giving an opinion on various indications of liver biopsy. There was a significant difference of opinion among residents and consultants as regards the indication of liver biopsy in coexisting conditions causing steatohepatitis $(p=0.006)$.

\section{Discussion}

Physicians are the first level of contact for most patients with NAFLD or patients with risk factors of NAFLD. Knowledge, awareness and attitude of these physicians are important for screening, diagnosing and encouraging these patients to adopt a healthy lifestyle and sustain it for a long period. The available studies indicate that physicians' awareness of NAFLD diagnosis and management was restricted and inadequate $[23,24]$. The gap in awareness and perception of these practitioners can be a major hurdle in optimising patient care. The present study demonstrates facts about the awareness and perception of consultant 
physicians and resident doctors of internal medicine about NAFLD.

\section{Awareness about prevalence}

A study conducted in last decade in West Bengal state of India revealed that NAFLD is prevalent among individuals in a developing country who, at cursory glance, may not appear to harbour the typical metabolic risk factors for NAFLD and may, therefore, be inappropriately perceived not to be at risk for the condition [14]. This can lead to gross underdiagnosis of the condition.

$70.8 \%$ of the participants of the present study indicated that the prevalence of NAFLD in India is in the range $20-40 \%$ which is in line with the reports about the global prevalence of NAFLD using different methods for diagnosis of NAFLD [25-31]. Incidence of this disease is expected to rise further in future with more urbanisation, socioeconomic growth, lack of health awareness, sedentary lifestyle and unhealthy dietary habits [32]. This study shows that $55 \%(n=132)$ of participants think that NAFLD can be a major health hazard in the country. Around $45 \%$ of participants including many who knew the prevalence and complications correctly, did not consider NAFLD as a major health problem. This is worrisome because the insufficient attention given to the risk factors by these physicians can lead to lethal underdiagnosis of the condition.

\section{Awareness about risk factors and association}

In this study, physicians and resident doctors (> 95\%) were well aware regarding the risk factors for developing NAFLD (diabetes mellitus, hypertension, obesity and dyslipidaemia) and awareness regarding associated complications such as apnoea, ischaemic heart disease, hypothyroidism, polycystic ovarian syndrome, etc. was lacking $(<60 \%)$, although the awareness level of the consultant physicians was better than the resident doctors. This difference might be due to the difference in clinical exposure to these types of patients and years of continued medical education in consultants. A study about awareness and attitude conducted in Wisconsin had shown higher knowledge about these associations even in primary care physicians in the USA [33]. A study from Sri Lanka had shown comparable results, but in the cohort of primary as specialist doctors [22].

\section{Awareness about diagnostic modalities}

The prevalence varies according to the diagnostic method. Although liver biopsy remains the gold standard for diagnosis of NAFLD/NASH [34], non-invasive imaging by ultrasonography, MRI, and thromboelastometry are sensitive modalities for diagnosing fatty liver. These modalities do not differentiate between steatosis and steatohepatitis [35-37]. Disagreement exists, however, regarding the necessity for performing liver biopsy in NAFLD patients. The recognition of appropriate clinical factors in conjunction with characteristic ultrasound findings can lead to an accurate diagnosis of NAFLD without the use of invasive testing, and also identify those individuals who should go on to have a liver biopsy [38]. Going by the current practice guidelines from the European Association for the Study of the Liver quoted earlier in the introduction, liver biopsy, ${ }^{1} \mathrm{H}-\mathrm{MRS}$ or MRI is necessary to diagnose a case of NAFLD [7].

Consultants in our study have unanimously vouched for ultrasound as the first and foremost investigation in NAFLD $(97 \%)$ as against residents $(78.3 \%)(p<0.0001)$. After USG, liver enzymes (94.4\%) followed by liver biopsy $(88.3 \%)$ were the investigations preferred by consultants, MRI (52.8\%) and thromboelastometry (21.1\%). Comparatively, residents advocated all these investigations in lower percentages. The plausible explanation of this is the basic barrier of confidence among residents in ordering higher investigations.

Figure 2 depicts the indications of liver biopsy as perceived by our participants. When the conditions causing steatohepatitis cannot be excluded, liver biopsy was advocated most by both groups, as against the situations when such conditions coexist. This contradiction was due to skewing due to less advocacy of liver biopsy by the resident group $(p=0.006)$. A plausible explanation is that such invasive investigations in conditions such as NAFLD are advised by consultants with proper comparative assessment of benefit outweighing risk of performing them. There was no significant difference in junior and senior consultants' advice.

\section{Perception about management}

A vast majority of both groups of participants agreed that diet (99.6\%), exercise (99.6\%) and avoidance of alcohol consumption $(91.3 \%)$ were the mainstay treatment options for NAFLD. However, there was no consensus on the percentage of weight loss and the health benefits. A modest weight loss of 5-10\% can improve the NAFLD activity score (NAS) and a weight loss of more than $10 \%$ reverses even fibrosis at least by one stage [39]. More than half of the physicians lacked this knowledge. Even in the diet advice, there was discrepancy as only $45 \%$ and $43 \%$ of participants overall advocated use of low lipid and hypocaloric diet respectively as a part of diet advice though the majority (99.6\%) agreed that 
diet advice is a mainstay of managing NAFLD. This was a poor practice of physicians observed in our study.

Among the other proposed medications, antioxidants were the most advocated, significantly more by consultants $(64.6 \%)$ than residents (51.7\%) $(p=0.016)$. Avoidance of hepatotoxic drugs was advised by $60.8 \%$ of participants of both groups. A comparatively lesser tendency to prescribe therapeutic agents such as vitamin E, pioglitazone and obeticholic acid was observed in both groups.

A study in tertiary care hospitals in Brisbane reported a striking finding that $71 \%$ of specialists in other branches reported that they made no referrals to hepatology services for an opinion regarding suspected NAFLD/NASH. The proposed explanation for this was that the majority of specialists $(>74 \%)$ did not believe that specific liver-directed pharmacologic therapy was involved in the therapeutic management of simple steatosis or NASH, and liver biopsy was not favoured as the most effective way to monitor patients with NAFLD for disease progression [24]. Our study, though focused on internal medicine practitioners, showed that only $22.8 \%$ of participants were referred to gastroenterologist/hepatologists. This is worrisome in view of optimal management with continuously evolving therapeutic guidelines which are more likely to be known to hepatologists than physicians.

\section{Barriers in the management of NAFLD}

Studies in literature which have evaluated barriers in the management of NAFLD have found that lack of confidence is the main barrier in different subject groups. A study on primary care physicians in Wisconsin, USA showed this as the most common barrier with $58 \%$ voting for it [33]. But, as a matter of worry, our study has shown this proportion to be much higher, $88.3 \%$, with an alarming fact that medicine specialists were our subjects. This can be attributed to inadequate update options of standard guidelines and protocols in developing countries even in this era. Cost of evaluation and treatment was the second most reported barrier among our participants, consultants reporting it significantly more $(73.3 \%)$ than residents $(58.7 \%)$ $(p=0.039)$. This again is attributable to lack of reasonably priced investigating modalities and overall lower socioeconomic status of the patients in developing countries. Other barriers such as time constraint and lack of compliance by patients were reported on smaller scales by both groups, $28.3 \%$ and $10 \%$ respectively.

\section{Awareness about NAFLD precipitating agents}

Figure 1 shows the awareness about worsening agents of NAFLD. Statins (93.3\%) were the most commonly per- ceived worsening agent, followed by paracetamol (85\%), azathioprine (82.5\%) and methotrexate (80.4\%). There was a statistically significant difference between the two groups, in the perception about the worsening effect of methotrexate, valproate, tamoxifen and amiodarone.

To summarise, our participants from both groups, resident doctors and consultant physicians, knew equally well about the association of NAFLD with diabetes and obesity, diet advice, usefulness of thromboelastometry and liver biopsy, dietary modification and exercise, usage of vitamin E, pioglitazone, statins and avoidance of hepatotoxic drugs and alcohol.

Consultants knew significantly better than residents about the prevalence of NAFLD, the associations and risk factors such as hypertension, dyslipidaemia, sleep apnoea. hypothyroidism, PCOS and IHD, non-invasive diagnostic modalities such as USG, MRI and liver enzymes, management options such as obeticholic acid, weight loss advice, and referral to a gastroenterologist. Resident doctors advocated use of antioxidants more than consultants.

\section{Strengths and limitations of the study}

The previously published studies have concentrated on a particular group of healthcare professionals with the same level of training and experience. The main strength of our study was the extensive coverage of medicine specialist doctors at different levels of training and practice. This helped us in better comparative assessment of knowledge, attitude and practices in the management of NAFLD. The sample size is a large representation of actual ultimate care givers.

The limitation while implementing the study was inadequate coverage of the entire spectrum of NAFLD in the questionnaire. The particular emphasis on the spectrum of NAFLD including NASH and progression to cirrhosis end stage liver failure would have been more corroborative.

\section{Conclusions}

Since there are many controversies and discrepancies in the literature regarding NAFLD, we have tried to impartially analyse the knowledge, attitude and practices of physicians in a cosmopolitan city.

This study highlights the need to increase widespread awareness programmes in the understanding of NAFLD and its major risk factors for physicians. It will aid in timely diagnosis and translate to early referrals to specialist doctors in order to have a positive impact on these patients' health. Furthermore, there is a need to educate and update doctors about the recent practice 
guidelines for NAFLD published by various societies in addition to highlighting the issues related to NAFLD in conferences, CMEs and peer group meetings.

\section{Acknowledgements}

We have modified the questionnaire devised by A.T. Matthias and team in Sri Lanka [22]. We would like to thank them for that.

We would like to extend our acknowledgements to Dr. Harshad Devarbhavi (Professor and Head Department of Gastroenterology and Hepatology, St. John's Medical College, Bangalore) for his expert review of the article and valuable suggestions.

\section{Disclosure}

The authors declare no conflict of interest.

\section{References}

1. Tai FW, Syn WK, Alazawi W. Practical approach to non-alcoholic fatty liver disease in patients with diabetes. Diabet Med 2015; 32: 1121-1133.

2. Mantovani A, Byrne CD, Bonora E, et al. Nonalcoholic fatty liver disease and risk of incident type 2 diabetes: a meta-analysis. Diabetes Care 2018; 41: 372-382.

3. Mantovani A, Zaza G, Byrne CD, et al. Nonalcoholic fatty liver disease increases risk of incident chronic kidney disease: a systematic review and meta-analysis. Metabolism 2018; 79: 64-76.

4. Targher G, Byrne CD, Lonardo A, et al. Non-alcoholic fatty liver disease and risk of incident cardiovascular disease: a meta-analysis. J Hepatol 2016; 65: 589-600.

5. Ekstedt M, Hagström H, Nasr P, et al. Fibrosis stage is the strongest predictor for disease-specific mortality in NAFLD after up to 33 years of follow-up. Hepatology 2015; 61: 1547-1554.

6. Söderberg C, Stål P, Askling J, et al. Decreased survival of subjects with elevated liver function tests during a 28 -year follow-up. Hepatology 2010; 51: 595-602.

7. EASL-EASD-EASO Clinical Practice Guidelines for the management of non-alcoholic fatty liver disease. Obesity Facts 2016; 9: 65-90.

8. Targher G, Day CP, Bonora E. Risk of cardiovascular disease in patients with nonalcoholic fatty liver disease. N Engl J Med 2010; 363: 1341-1350.

9. Targher G, Lonardo A, Byrne CD. Nonalcoholic fatty liver disease and chronic vascular complications of diabetes mellitus. Nat Rev Endocrinol 2018; 14: 99-114.

10. Allen AM, Ray Kim W, Moriarty JP, et al. Nonalcoholic fatty liver disease incidence and impact on metabolic burden and death: a 20 year-community study. Hepatology 2016; 64: 2165-2172.

11. Amarapurkar D, Kamani P, Patel N, et al. Prevalence of non-alcoholic fatty liver disease: population-based study. Ann Hepatol 2007; 6: 161-163.

12. Younossi Z, Anstee Q, Marietti M, et al. Global burden of NAFLD and NASH: trends, predictions, risk factors and prevention. Nat Rev Gastroenterol Hepatol 2018; 15: 11-20.

13. Chalasani N, Younossi Z, Lavine JE, et al.; American Gastroenterological Association; American Association for the Study of Liver Diseases; American College of Gastroenterologyh. The diagnosis and management of non-alcoholic fatty liver disease: practice guideline by the American Gastroenterological Association, American Association for the Study of Liver Diseases, and American College of Gastroenterology. Gastroenterology 2012; 142: 1592-1609.

14. Das K, Das K, Mukherjee PS, et al. Nonobese population in a developing country has a high prevalence of nonalcoholic fatty liver and significant liver disease. Hepatology 2010; 51: 1593-1602.

15. Singh SP, Nayak S, Swain M, et al. Prevalence of nonalcoholic fatty liver disease in coastal eastern India: a preliminary ultrasonographic survey. Trop Gastroenterol 2004; 25: 76-79.

16. Anurag L, Aniket S, Shalik J, et al. Non-alcoholic fatty liver disease prevalence and associated risk factors - a study from rural sector of Maharashtra. Trop Gastroenterol 2015; 36: 25-30.

17. Pais R, Charlotte F, Fedchuk L, et al.; LIDO Study Group. A systematic review of follow-up biopsies reveals disease progression in patients with non-alcoholic fatty liver. J Hepatol 2013; 59: 550-556.

18. McPherson S, Hardy T, Henderson E, et al. Evidence of NAFLD progression from steatosis to fibrosingsteatohepatitis using paired biopsies: implications for prognosis and clinical management. J Hepatol 2015; 62: 1148-1155.

19. Younossi ZM, Otgonsuren M, Henry L, et al. Association of nonalcoholic fatty liver disease (NAFLD) with hepatocellular carcinoma (HCC) in the United States from 2004 to 2009. Hepatology 2015; 62: 1723-1730.

20. Mittal S, El-Serag HB, Sada YH, et al. Hepatocellular carcinoma in the absence of cirrhosis in United States veterans is associated with nonalcoholic fatty liver disease. Clin Gastroenterol Hepatol 2016; 14: 124-131.e1.

21. Lavekar A, Satyanarayana P, Lavekar A. How is silent 'non-alcoholic fatty liver disease' epidemic perceived in India? A community based cross sectional study. Int J Commun Med Public Health 2019; 6: 5256-5261.

22. Matthias AT, Fernandopulle ANR, Seneviratne SL. Survey on knowledge of non-alcoholic fatty liver disease (NAFLD) among doctors in Sri Lanka: a multicenter study. BMC Res Notes 2018; 11: 556 .

23. Grattagliano I, D’Ambrosio G, Palmieri VO, et al. Improving nonalcoholic fatty liver disease management by general practitioners: a critical evaluation and impact of an educational training program. J Gastrointestin Liver Dis 2008; 17: 389-394.

24. Bergqvist CJ, Skoien R, Horsfall L, et al. Awareness and opinions of non- alcoholic fatty liver disease by hospital specialists. Intern Med J 2013; 43: 247-253.

25. Farrell GC, Larter CZ. Nonalcoholic fatty liver disease: from Steatosis to cirrhosis. Hepatology 2006; 43 (Suppl. 1): S99-112.

26. McCullough AJ. The epidemiology and risk factors of NASH. In: Farrell GC, George J, de la M Hall P, McCullough AJ (Eds.). Fatty liver disease: NASH and related disorders. Blackwell Publishing, Malden, MA 2005; 23-37.

27. Weston SR, Leyden W, Murphy R, et al. Racial and ethnic distribution of nonalcoholic fatty liver in persons with newly diagnosed chronic liver disease. Hepatology 2005; 41: 372-379.

28. Agarwal SR, Malhotra V, Sakhuja P, Sarin S. Clinical biochemi$\mathrm{cal}$ and histological profile of nonalcoholic steatohepatitis. Indian J Gastroenterol 2001; 20: 183-186.

29. Madan K, Batra Y, Panda S, et al. Role of polymerase chain reaction and liver biopsy in the evaluation of patients with asymptomatic transaminitis. Implications in diagnostic approach. J Gastroenterol Hepatol 2004; 20: 1291-1299.

30. Singh S, Kuftinec GN, Sarkar S. Non-alcoholic fatty liver disease in South Asians: a review of the literature. J Clin Transl Hepatol 2017; 5: 76-81. 
31. Mohan V, Farooq S, Deepa M, et al. Prevalence of nonalcoholic fatty liver disease in urban south Indians in relation to different grades of glucose intolerance and metabolic syndrome. Diabetes Res Clin Pract 2009; 84: 84-91.

32. Singh SP, Singh A, Misra D, et al. Risk factors associated with non-alcoholic fatty liver disease in Indians: a case-control study. J Clin Exp Hepatol 2015; 5: 295-302.

33. Said A, Gagovic V, Malecki K, et al. Primary care practitioners survey of non-alcoholic fatty liver disease. Ann Hepatol 2013; 12: 758-765.

34. Kleiner DE, Brunt EM, Van Natta M, et al. Design and validation of a histological scoring system for non-alcoholic fatty liver disease. Hepatology 2005; 41: 1313-1321.

35. Gore RM. Diffuse liver disease. In: Gore RM, Levine MS, Laufer I (Eds.). Textbook of gastrointestinal radiology. Saunders, Philadelphia 1994; 1968-2017.

36. Yajima Y, Ohta K, Narui T, et al. Ultrasonographical diagnosis of fatty liver: significance of the liver-kidney contrast. Tohoku J Exp Med 1983; 139: 43-50.

37. Fusamoto H, Suzuki K, Hayashi N, et al. Obesity and liver disease: evaluation of fatty infiltration of the liver using ultrasonic attenuation. J Nutr Sci Vitaminol Tokyo 1991; 37 (Suppl.): S71-77.

38. Khov N, Sharma A, Riley TR. Bedside ultrasound in the diagnosis of nonalcoholic fatty liver disease. World J Gastroenterol 2014; 20: 6821-6825.

39. Romero-Gómez M, Zelber-Sagi S, Trenell M. Treatment of NAFLD with diet, physical activity and exercise. J Hepatol 2017; 67: 829-846. 